\title{
The potential for vaccination-induced herd immunity against the SARS-CoV-2 B.1.1.7 variant
}

David Hodgson ${ }^{1}$, Stefan Flasche ${ }^{1}$, Mark Jit' ${ }^{1}$, Adam J Kucharski' ${ }^{1}$, CMMID COVID-19 Working Group ${ }^{1,2}$

1. Centre for Mathematical Modelling of Infectious Diseases, London School of Hygiene \& Tropical Medicine, London, United Kingdom

2. The members of the Working Group are listed below

Correspondence: David Hodgson (david.hodgson@lshtm.ac.uk)

Investigators: The investigators list are listed at the end of the article

Citation style for this article:

Hodgson David, Flasche Stefan, Jit Mark, Kucharski Adam J, CMMID COVID-19 Working Group. The potential for vaccination-induced herd immunity against the SARS-CoV-2 B.1.1.7 variant. Euro Surveill. 2021;26(20):pii=2100428. https://doi.org/10.2807/1560-7917.ES.2021.26.20.2100428

We assess the feasibility of reaching the herd immunity threshold against SARS-CoV-2 through vaccination, considering vaccine effectiveness (VE), transmissibility of the virus and the level of pre-existing immunity in populations, as well as their age structure. If highly transmissible variants of concern become dominant in areas with low levels of naturally-acquired immunity and/or in populations with large proportions of $<15$ year-olds, control of infection without non-pharmaceutical interventions may only be possible with a $\mathrm{VE} \geq 80 \%$, and coverage extended to children.

Initial reports of vaccine effectiveness against severe acute respiratory syndrome coronavirus 2 (SARSCoV-2), the virus responsible for coronavirus disease (COVID-19), have suggested a substantial reduction of the risk of infection [1]. Nevertheless, with the emergence of more transmissible variants such as B.1.1.7 [2], how large-scale immunisation programmes against SARS-CoV-2 will perform is currently unclear. This study assesses the potential of COVID-19 vaccination to generate herd immunity and takes into account vaccine effectiveness, naturally-acquired immunity and achievable vaccination coverage (depending on the population age structure), as well as two transmissibility scenarios ((i) with pre-B.1.1.7, and (ii) with exclusively B.1.1.7 variants).

\section{Vaccination and herd immunity}

The feasibility of attaining vaccination-induced herd immunity depends on (i) vaccine effectiveness in reducing transmission, (ii) the transmissibility of the target pathogen and (iii) the vaccine coverage that is achievable in a population.

In a scenario where vaccines are distributed randomly across a population, the herd immunity threshold (HIT) for an immunisation programme is defined as $1-1 / R_{0}$, where $R_{0}$ is the basic reproduction number [3]. Note that if $R_{0}$ is calculated using an age- or risk-structured next generation matrix then this equation will still hold. If vaccine effectiveness is below the HIT, then even vaccination of the entire population would, on its own, be insufficient to ensure control (i.e. the effective reproduction number, accounting for immunity, would remain above 1 ).

\section{Herd immunity, vaccine effectiveness and virus transmissibility}

Viruses with vaccines of varying effectiveness and different transmissibility

Comparing this theoretical HIT with estimated values of $R_{0}$ and vaccine effectiveness for a range of vaccinepreventable diseases (Figure 1), we see that for infections caused by viruses with little antigenic variation, vaccine effectiveness is sufficiently high to control transmission if high vaccine coverage is achieved. This is why, in many countries, childhood immunisation programmes have led to elimination of viruses with little antigenic variation and long-lasting sterilizing immunity, such as measles and rubella viruses [4].

In contrast, viruses that undergo frequent antigenic turnover, such as influenza viruses necessitate regular vaccine updates and re-vaccination [5]. Seasonal influenza vaccine effectiveness is influenced by antigenic evolution of influenza viruses, with similar rates of evolution to that observed for seasonal human coronaviruses. Moreover, the effectiveness depends on whether the influenza vaccine strains are or not the same as the circulating ones. In the event of well-matched influenza vaccines, the effectiveness may nevertheless still be below the HIT. For influenza $A\left(\mathrm{H}_{3} \mathrm{~N}_{2}\right)$ virus, for example, the estimated effectiveness of an antigenically-matched vaccine (33\%; $95 \%$ confidence interval (Cl):22-43) [6] implies that control of this subtype in 
Comparison of effectiveness of currently available vaccines against herd immunity threshold for different viruses, 20002021 ( $n=9$ virus variants/types/subtypes)

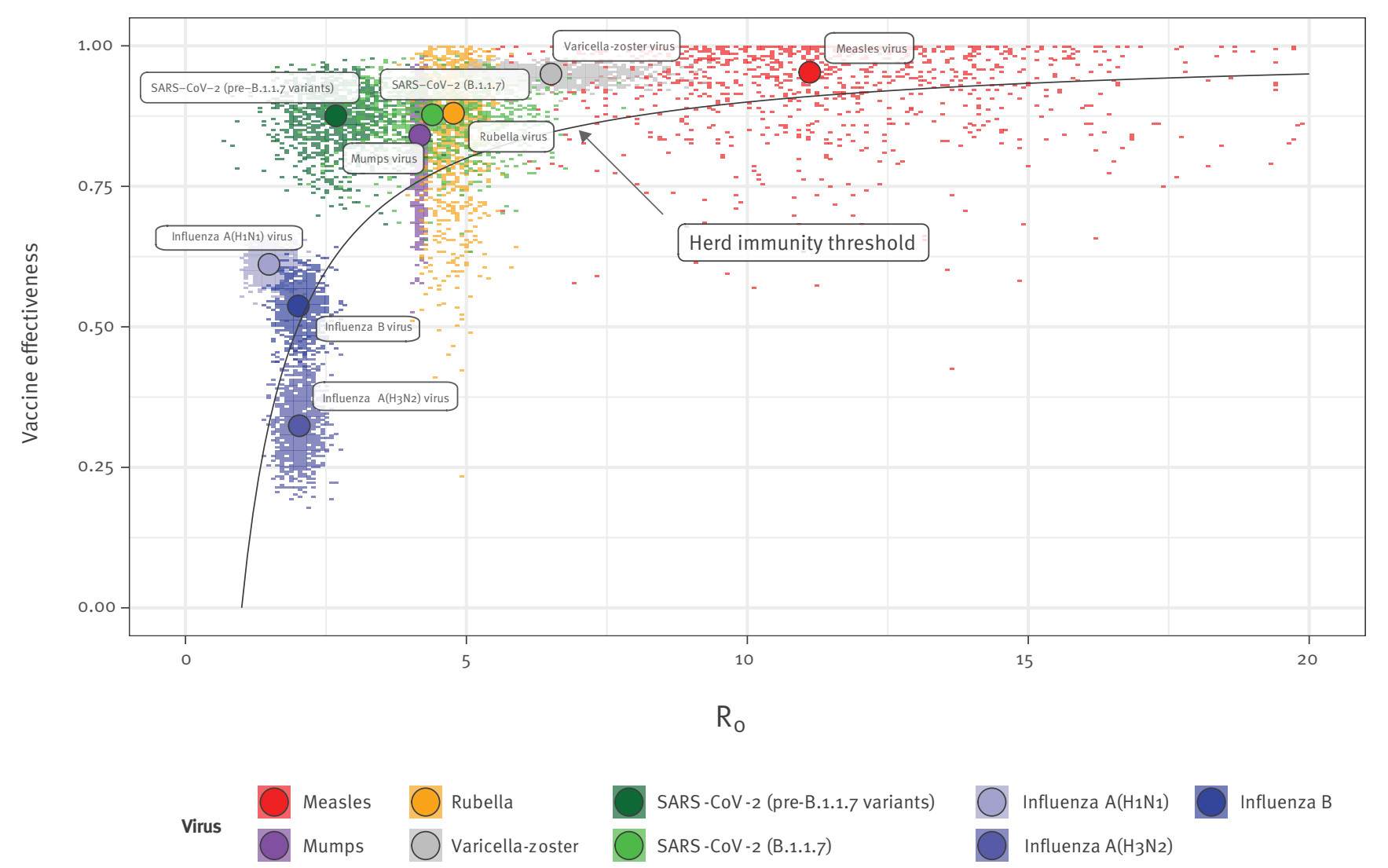

SARS-CoV-2: severe acute respiratory syndrome coronavirus 2.

The black line shows the minimum vaccine effectiveness needed to achieve herd immunity for given $\mathrm{R}$ values. Colour points represent samples from available effectiveness and transmissibility estimates (see Supplement), with large circles showing medians. If sampled points are above the line, vaccination of the entire population could in theory lead to epidemic control; the more samples that are above the line, the higher the probability of control.

the absence of natural immunity is unlikely, even in theory; we estimate a very small probability (defined as number of Monte Carlo samples) in an unexposed population with $100 \%$ vaccination coverage of being above the HIT.

\section{SARS-CoV-2}

For SARS-CoV-2, we consider two SARS-CoV-2 variants for which we assume vaccination provides equal protection: pre-B.1.1.7 variants with an $R_{0}$ of 2.7 (95\% Cl: 1.5-3.8) and the B.1.1.7 variant with an $\mathrm{R}_{0}$ of 4.5 (95\% Cl:2.5-6.4) [7]. Assuming 86\% (95\% Cl:76-97) vaccine effectiveness against infectiousness, based on early estimates of protection against infection following two doses of Comirnaty (BNT162b2, BioNTech/ Pfizer, Mainz, Germany/New York, United States) [1], we estimate, in the case of a pre-B.1.1.7 variant, a 99\% probability of being above the HIT with whole-population coverage and a $94 \%$ probability if B.1.1.7 is circulating exclusively.

\section{Ethical statement}

Ethical approval was not necessary for this modelling study as the analysis uses only aggregated secondary data from published articles.

\section{COVID-19 vaccination coverage achievable}

The estimated $94 \%$ and $99 \%$ probabilities to be above the HIT, for B.1.1.7 and pre-B.1.1.7 SARS-CoV-2 respectively, are based on the assumption that the whole population is vaccinated. However, whole-population vaccination would require SARS-CoV-2 vaccines which, as at mid-May 2021, are only approved for adults in most countries - to also be used at high coverage in children, for whom there is currently limited evidence from trials only, on safety or effectiveness.

Assuming a vaccination campaign aimed at all individuals $\geq 15$ years old, the proportion of the population currently eligible for vaccination (i.e. comprising people aged $\geq 15$ years) varies between countries, with the proportion of children within a country's population decreasing along increasing income brackets (low, 
Vaccination coverage required to reach herd immunity for COVID-19, considering three different levels of vaccine effectiveness in (A) pre-B.1.1.7 and (B) B.1.1.7 SARS-CoV-2 transmission scenarios, 2021

A. Sars-Cov-2 (pre-B.1.1.7. variants)

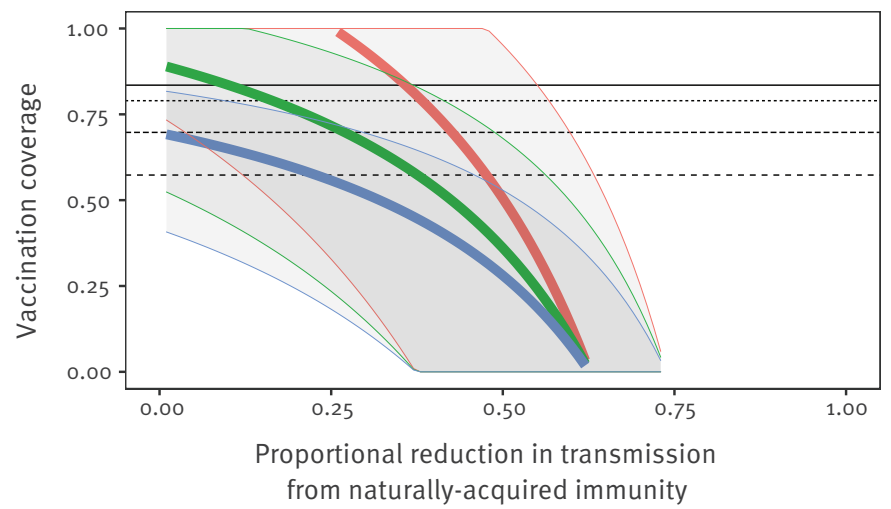

B. Sars-Cov-2 (B.1.1.7.)

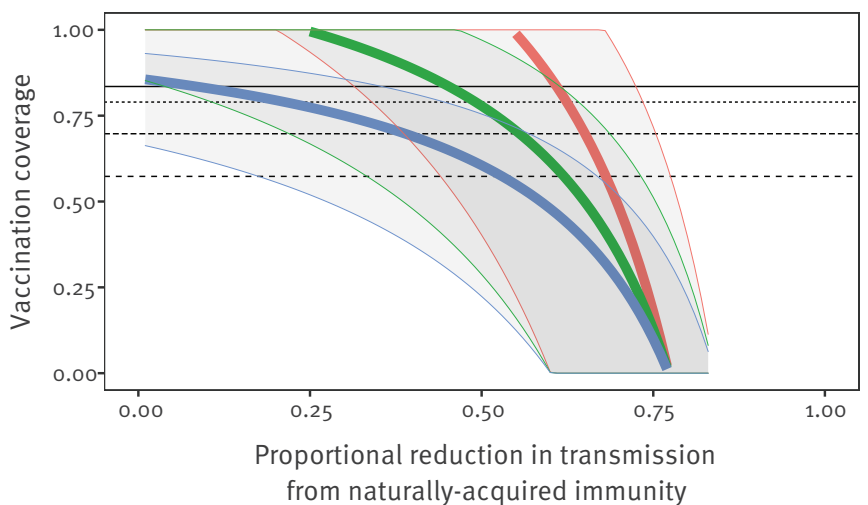

\author{
$\begin{array}{llll}\text { Vaccine effectiveness (\%) } \square 50 \quad \square & \square 0 \quad \square 90\end{array}$ \\ Proportion $\geq \mathbf{1 5}$ year-olds _ High income ..... Upper middle income -.- Lower middle income $\quad$ - .. Low income
}

COVID-19: coronavirus disease; Crl: credible intervals; SARS-CoV-2: severe acute respiratory syndrome coronavirus 2.

Vaccination coverage required to reach herd immunity considering different levels of vaccine effectiveness and naturally-acquired immunity for SARS-CoV-2 in (A) a pre-B.1.1.7 transmission scenario and (B) a B.1.1.7 one.

Data sources are provided in the Supplement. Thick coloured lines show medians and shaded regions $95 \% \mathrm{Crl}$. Blue is the vaccine coverage necessary to reduce transmission when vaccine effectiveness is $90 \%$; green, $70 \%$; red, $50 \%$. Black horizontal lines show the maximum vaccination coverage (i.e. when all people $\geq 15$ years old are vaccinated) for different groups of countries, according to the proportions of $\geq 15$ year-olds in their population, which are proxied by country income. The lower the country level of income, the lower the proportion of $\geq 15$ year-olds in its population is, as well as the maximum vaccine coverage achievable. Maximum achievable vaccination coverage is averaged across all countries in particular income groups.

lower-middle, upper-middle, upper income). Given this trend, we use income level as a proxy for the total proportion of the population aged 15 years and over, which is eligible for vaccination.

\section{COVID-19 vaccination coverage needed considering natural immunity}

Depending on vaccine coverage and effectiveness against infectiousness with future circulating variants - which may be antigenically dissimilar to B.1.1.7 [8] herd immunity to SARS-CoV-2 in the absence of other non-pharmaceutical interventions may not be reached until considerable natural immunity has accumulated. As some countries now have a sizeable subpopulation with protective antibodies acquired through natural infection [9], we estimated the probability of reaching the HIT for SARS-CoV-2 under varying degrees of vaccination coverage against a background of reduction in transmission from natural immunity.

\section{Pre-B.1.1.7 SARS-CoV-2 transmission scenario} We estimated that for pre-B.1.1.7 SARS-CoV-2 variants, an immunisation programme targeting all persons aged $\geq 15$ years (as would be the case for a vaccine not approved for use in younger groups), would have generated herd immunity against homotypic viruses in most higher income countries, regardless of the level of natural immunity, if vaccine effectiveness is at least $70 \%$ (or at least $90 \%$, with a high degree of confidence). However, the high proportion of children in many lower income countries means the HIT cannot be reached with $\mathrm{a} \geq 15$-years-old vaccination programme alone and would require higher levels of immunity among children, acquired either through vaccination or infection, to be reached (Figure $2 \mathrm{~A}$ ).

\section{SARS-CoV-2 B.1.1.7 transmissibility scenario}

For B.1.1.7, or similarly transmissible variants, we would expect ongoing transmission until a sufficient level of natural immunity has been accrued, even in countries with an older age distribution. In our results, the estimated level of natural immunity to reach the HIT alongside vaccination varied considerably across countries, with high-income countries, which have a high proportion of adults, needing a $10 \%$ reduction in transmission from natural immunity on average, alongside widespread vaccination of all persons $\geq 15$ yearsold, and low-income countries with a lower proportion of adults, needing a $53 \%$ reduction in transmission for the same vaccination programme (Figure $2 \mathrm{~B}$ ). 


\section{FIGURE 3}

Estimated seroprevalence and eligible proportion for vaccination in different geographical areas, in relation to the herd immunity thresholds obtained with different vaccine effectiveness, considering (A) pre-B.1.1.7 and (B) B.1.1.7 SARS-CoV-2 transmission scenarios, seroprevalence estimates are from September 2020-April 2021

A. Pre-B.1.1.7 variants

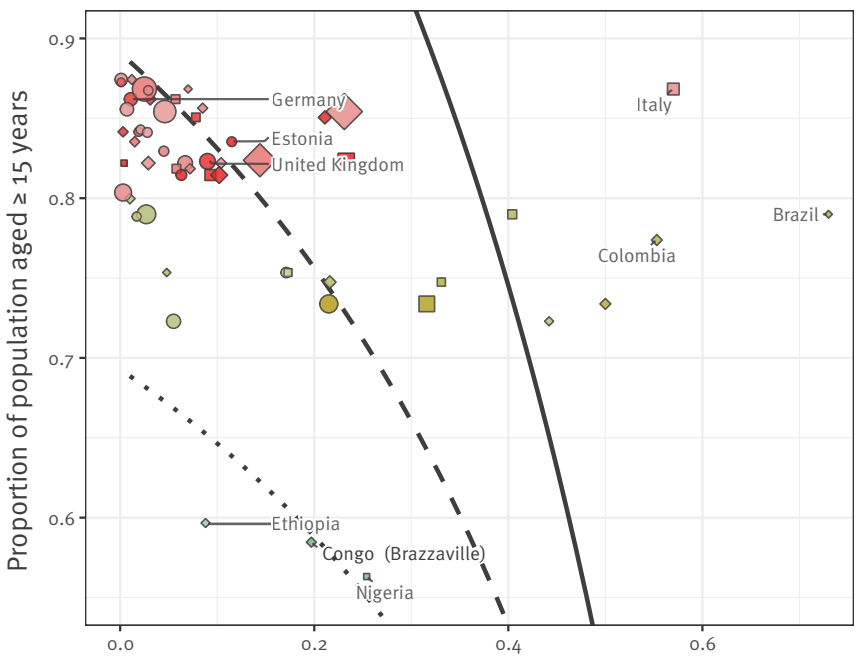

Estimated seroprevalence

\section{B. B.1.1.7 variants}

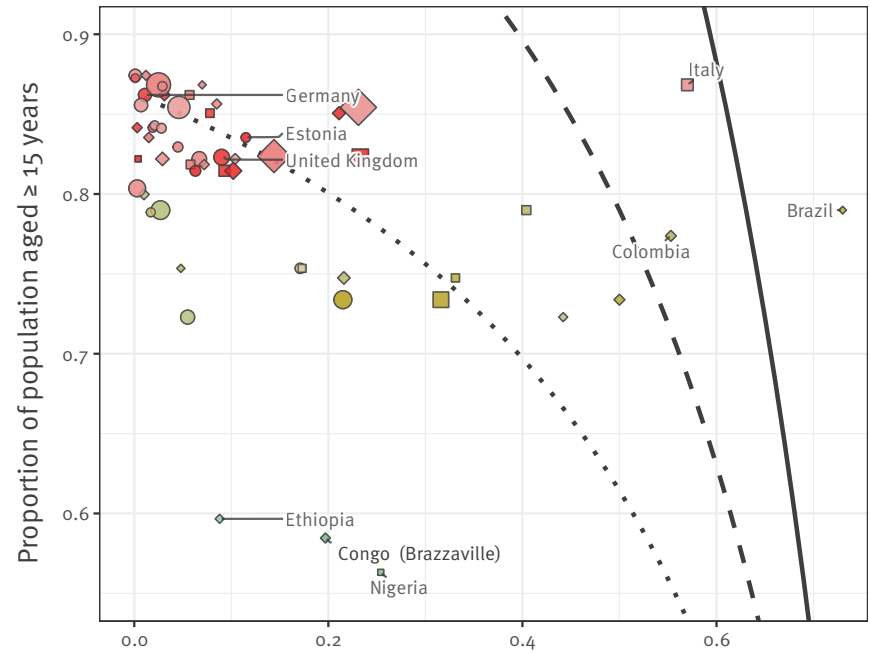

Estimated seroprevalence

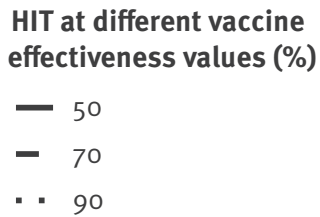

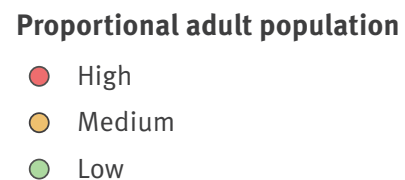

Proportional adult population

$\bigcirc$ Low

Adults: individuals aged $\geq 15$ years; HIT: herd immunity threshold; SARS-CoV-2: severe acute respiratory syndrome coronavirus 2 .

Panel (A) shows results considering pre-B.1.1.7 transmission while panel (B) shows results obtained with B.1.1.7 variant transmissibility.

By assuming that the estimated seroprevalence is equal to the proportional decrease in transmission because of natural immunity and that the proportion of adults is equal to the maximum achievable vaccination coverage, the plots show how feasible it is for various areas in the world to reach herd immunity against pre-B.1.1.7 or B.1.1.7 variants after a mass vaccination programme, for various vaccine effectiveness values.

The shape of the point marker represents the geographical scope of population used for the seroprevalence [12] estimate (national, regional or local) and the point marker radius scales with size of the study population.

The size of the point markers is proportional to the study size of the serosurvey.

The proportion of the population who are adults in the study site is given by colour hue: high category is above $80 \%$ (red), medium category is between 70 and $80 \%$ (orange), and low category is less than $70 \%$ (green) and the time since sample collection finished is shown through colour saturation, (i.e. high saturated points represent studies which were conducted more recently). The black line-types show the HIT thresholds for different vaccine coverage values. Seroprevalence data from serotracker.com.

\section{SARS-CoV-2 seroprevalence as a proxy for natural immunity}

We can use regional estimates of seroprevalence to approximate the proportional reduction in SARS-CoV-2 transmission due to natural immunity. For simplicity, we assume random mixing within a population, seropositivity as a perfect marker of immunity, and that natural immunity is fully sterilising (stops onward transmission). This is based on observations that seropositivity is associated with at least a $0.84(95 \% \mathrm{Cl}$ :
$0.81-0.87$ ) reduction in risk of reinfection [10], and reinfections are likely to in turn be less infectious [11]. As seropositivity from natural infection is likely to be concentrated among groups more involved in transmission (i.e. seroprevalence would underestimate the corresponding reduction in transmission) this would also offset any contribution to transmission from individuals who have immunity that is not fully sterilising. 
Controlling SARS-CoV-2 spread in populations with different age structure and seroprevalence By assuming that the maximum vaccination coverage feasible is equal to the proportion of the population aged $\geq 15$ years, we can therefore assess the feasibility in countries with varying proportions of adult population of reaching the HIT (Figure 3).

We obtain seroprevalence estimates from SeroTracker [12], and population distribution estimates from The World Bank [13].

\section{Pre-B.1.1.7 SARS-CoV-2 transmissibility scenario}

When pre-B.1.1.7 SARS-CoV-2 variants circulate, countries with a high proportion of persons $\geq 15$ years old could theoretically reach the HIT through an adult mass immunisation programme with a vaccine effectiveness as low as $70 \%$. For countries with a lower proportion of persons $\geq 15$ years old, our results suggest adult immunisation campaigns alone would not be sufficient to provide vaccine-induced herd immunity if children contribute equally to transmission.

\section{SARS-CoV-2 B.1.1.7 transmissibility scenario}

If the B.1.1.7 variant dominates, countries with a high proportion of adults require much higher effectiveness, ca $90 \%$, to reach the HIT after an adult immunisation campaign, and many countries with a lower adult population proportion are again unlikely to reach the HIT through vaccination. Although there are some regional and local studies in countries such as Brazil [14], Italy [15], and Columbia [9] showing high seroprevalence at the local level, much lower seroprevalence has been estimated at the national level. For countries in which there is substantial heterogeneity in natural immunity, these estimates for herd immunity are unlikely to equate to a pragmatic protective threshold. However, estimates for herd immunity in such areas could be improved by developing models that include contact structure and geospatial clustering.

\section{Discussion and conclusion}

This study considers the feasibility of reaching the herd immunity threshold against SARS-CoV-2 through vaccination and draws comparisons with other vaccine-preventable pathogens, including influenza and common immunising childhood infections.

Our observations suggest that if highly transmissible variants become dominant in areas with low seroprevalence, and/or in populations with a high proportion of children, control of infection by vaccination, in the absence of non-pharmaceutical interventions, may only be achievable with a vaccine effectiveness against infectiousness of $\geq 80 \%$ - as suggested by early data for the Comirnaty vaccine [1] - or next generation vaccines with persistently high effectiveness and crossprotection against antigenic variants, extended to the full population, including children.
As further vaccine effectiveness data emerge, our estimates of the potential for vaccination-induced-control could be further refined. Local differences in population age structure and behaviour, as well as biological characteristics of SARS-CoV-2 variants, could also change both baseline transmissibility between countries and which groups drive outbreaks [16]. If vaccine impact in reducing transmission is in reality higher than assumed here, the feasibility of local elimination would increase; for example, there is emerging evidence that infected vaccinees have a reduced risk of onward transmission (see Supplement). Conversely, future variants could reduce the effectiveness of current COVID-19 vaccines, and theoretical potential for local elimination, much as influenza vaccines are less effective against heterotypic strains [17]. In particular, while a highly transmissible homotypic SARS-CoV-2 variant would increase the herd immunity threshold, but to a value still below $100 \%$, a sufficiently antigenically variable variant would mean herd immunity cannot be achieved with existing vaccines.

In the absence of booster campaigns and expanded coverage, herd immunity reached through a combination of vaccination and natural immunity could be short-lived. Therefore, our estimates are likely to be optimistic, as vaccine impact would decline as a result of increasing susceptibility from new births, waning protection and antigenic evolution (Supplemental Figure S1). However, some countries are considering third doses following their initial vaccination campaigns, which may counteract these effects [18].

As at mid-May 2021, vaccines are only approved for adults in most countries. If evidence on vaccine safety or effectiveness in children emerges from trials, the acceptability of vaccinating children, for whom an estimated 0.1 to $0.3 \%$ of symptomatic cases result in hospitalisation, may in practice be very different to adults, where between 1 and $30 \%$ of symptomatic cases result in hospitalisation, depending on age [19]. However, if such programmes are deemed feasible, overall vaccination impact could be increased if uptake is high among younger groups, such as young adults or older children, who contribute most to transmission [20].

In conclusion, based on current evidence, when considering reopening strategies, policymakers in countries with low seroprevalence or a high proportion of children should not assume that even vaccination of all adults will be sufficient to reach the herd immunity threshold. However, vaccination could still dramatically reduce the impact of SARS-CoV-2 infection on resulting disease within a population, particularly among groups at higher risk. This emphasises the importance, particularly in regions with limited vaccine-rollout capabilities and low seroprevalence, of public health measures and vaccination campaigns focused on reducing future COVID-19 disease burden, instead of relying on an assumption that transmission will necessarily be eliminated through vaccination. 


\section{Investigators}

Centre for Mathematical Modelling of Infectious Disease (CMMID) COVID-19 Working Group: Sam Abbott, W John Edmunds, Nicholas G. Davies, Rosalind M Eggo, Graham Medley, Jiayao Lei, Yang Liu, Damien C Tully, Ciara V McCarthy, Paul Mee, Akira Endo, Joel Hellewell, Sebastian Funk, Thibaut Jombart, Yalda Jafari, Oliver Brady, Kiesha Prem, Fabienne Krauer, Mihaly Koltai, Naomi R Waterlow, Timothy W Russell, Sophie R Meakin, Kathleen O'Reilly, Nikos I Bosse, William Waites, Emily S Nightingale, Rachel Lowe, Yung-Wai Desmond Chan, Katherine E. Atkins, Billy J Quilty, Frank G Sandmann, Kevin van Zandvoort, C Julian Villabona-Arenas, Hamish P Gibbs, James D Munday, Anna M Foss, Amy Gimma, Carl A B Pearson, Rosanna C Barnard, Matthew Quaife, Fiona Yueqian Sun, Alicia Rosello, Rachael Pung, Christopher I Jarvis, Emilie Finch, Kaja Abbas, Samuel Clifford, Gwenan M Knight, Simon R Procter

\section{Code availability}

The datasets generated during and analysed during the current study are available in the adamkucharski/hit-analysis repository, https://github.com/adamkucharski/hit-analysis.

\section{Acknowledgements}

The following funding sources are acknowledged as providing funding for the working group authors. This research was partly funded by the Bill \& Melinda Gates Foundation (INV-001754: MQ; INV-003174: JYL, KP, YL; INV-016832: SRP; NTD Modelling Consortium OPP1184344: CABP, GFM; OPP1139859: BJQ; OPP1183986: ESN; OPP1191821: KO'R). BMGF (INV-016832; OPP1157270: KA). CADDE MR/S0195/1 \& FAPESP 18/14389-0: PM. EDCTP2 (RIA2020EF-2983-CSIGN: HPG). Elrha R2HC/UK FCDO/Wellcome Trust/This research was partly funded by the National Institute for Health Research (NIHR) using UK aid from the UK Government to support global health research. The views expressed in this publication are those of the author(s) and not necessarily those of the NIHR or the UK Department of Health and Social Care: KvZ. ERC Starting Grant (\#757699: MQ). ERC (SG 757688: CJVA, KEA). This project has received funding from the European Union's Horizon 2020 research and innovation programme - project EpiPose (101003688: AG, KP, RCB, WJE, YL). FCDO/Wellcome Trust (Epidemic Preparedness Coronavirus research programme 221303/Z/20/Z: CABP, KvZ). This research was partly funded by the Global Challenges Research Fund (GCRF) project 'RECAP' managed through RCUK and ESRC (ES/P010873/1: CIJ, TJ). HDR UK (MR/So03975/1: RME). HPRU (NIHR200908: NIB). Innovation Fund (01VSF18015: FK). MRC (MR/N013638/1: EF, NRW; MR/V027956/1: WW). Nakajima Foundation: AE. NIHR (16/136/46: BJQ; 16/137/109: BJQ, FYS, YL; Health Protection Research Unit for Modelling Methodology HPRU-2012-10096: TJ; NIHR200908: RME; NIHR200929: CVM, FGS, NGD; PR-OD-1017-20002: AR, WJE). Royal Society (Dorothy Hodgkin Fellowship: RL). Singapore Ministry of Health: RP. UK DHSC/UK Aid/NIHR (PR-OD-1017-20001: HPG). UK MRC (MC PC 19065 - Covid 19: Understanding the dynamics and drivers of the COVID-19 epidemic using real-time outbreak analytics: NGD, RME, SC, TJ, WJE, YL; MR/P014658/1: GMK). Authors of this research receive funding from UK Public Health Rapid Support Team funded by the United Kingdom Department of Health and Social Care: TJ. UKRI Research England: NGD. UKRI (MR/Vo28456/1: YJ). Wellcome Trust (206250/Z/17/Z: TWR; 206471/Z/17/Z: OJB; 208812/Z/17/Z: SC; 210758/Z/18/Z: JDM, JH, SA, SFunk, SRM; 221303/Z/20/Z: MK; UNS110424: FK). No funding: AMF, DCT, YWDC.

\section{Conflict of interest}

None declared.

\section{Authors' contributions}

A.K. devised the study, A.K and D.H. carried out the mathematical analysis. A.K, D.H, S.F and M.J contributed to the analysis of the results, the figure and to the writing of the manuscript. The manuscript was reviewed and checked by the Centre for Mathematical Modelling of Infectious Disease (CMMID) Working Group. Each member of the Working Group contributed in processing, cleaning and interpretation of data, interpreted findings, contributed to the manuscript, and approved the work for publication.

\section{References}

1. Hall VJ, Foulkes S, Ayoub Saei A, Andrews N, Oguti B, Charlett $A$, et al. Effectiveness of BNT162 b2 mRNA Vaccine Against Infection and COVID-19 Vaccine Coverage in Healthcare Workers in England, Multicentre Prospective Cohort Study (the SIREN Study). SSRN Electron J. 2021. https://doi.org/10.2139/ ssrn.3790399

2. Davies NG, Abbott S, Barnard RC, Jarvis Cl, Kucharski A] Munday JD, et al., CMMID COVID-19 Working Group, COVID-19 Genomics UK (COG-UK) Consortium. Estimated transmissibility and impact of SARS-CoV-2 lineage B.1.1.7 in England. Science. 2021;372(6538):eabg3055. https://doi.org/10.1126/science. abg3055 PMID: 33658326

3. Fine P, Eames K, Heymann DL. "Herd immunity": a rough guide. Clin Infect Dis. 2011;52(7):911-6. https://doi.org/10.1093/cid/ ciroo7 PMID: 21427399

4. World Health Organization (WHO). Feasibility Assessment of Measles and Rubella Eradication. Geneva: WHO; 2019. Available from: https://www.who.int/immunization/sage/ meetings/2019/october/Feasibility_Assessment_of_Measles_ and_Rubella_Eradication_final.pdf

5. Kistler KE, Bedford T. Evidence for adaptive evolution in the receptor-binding domain of seasonal coronaviruses $0 C_{43}$ and 229e. eLife. 2021;10:e64509. https://doi.org/10.7554/ eLife.64509 PMID: 33463525

6. Belongia EA, Simpson MD, King JP, Sundaram ME, Kelley NS, Osterholm MT, et al. Variable influenza vaccine effectiveness by subtype: a systematic review and meta-analysis of testnegative design studies. Lancet Infect Dis. 2016;16(8):94251. https://doi.org/10.1016/S1473-3099(16)00129-8 PMID: 27061888

7. Davies NG, Abbott S, Barnard RC, Jarvis Cl, Kucharski AJ, Munday JD, et al. , CMMID COVID-19 Working Group, COVID-19 Genomics UK (COG-UK) Consortium. Estimated transmissibility and impact of SARS-CoV-2 lineage B.1.1.7 in England. Science. 2021;372(6538): eabg3055. https://doi.org/10.1126/science. abg3055 PMID: 33658326

8. Garcia-Beltran WF, Lam EC, St. Denis K, Nitido AD, Garcia $\mathrm{ZH}$, Hauser BM, et al. Multiple SARS-CoV-2 variants escape neutralization by vaccine-induced humoral immunity. medRxiv. 2021; 2021.02.14.21251704. https://doi.org/http://dx.doi.org/ 10.1101/2021.02.14.21251704

9. Mattar S, Alvis-Guzman N, Garay E, Rivero R, García A, Botero $Y$, et al. Severe Acute Respiratory Syndrome Coronavirus 2 Seroprevalence Among Adults in a Tropical City of the Caribbean Area, Colombia: Are We Much Closer to Herd Immunity Than Developed Countries? Open Forum Infect Dis. 2020;7(12):ofaa550. https://doi.org/10.1093/ofid/ofaa550 PMID: 33354587

10. Hall VJ, Foulkes S, Charlett A, Atti A, Monk EJM, Simmons $\mathrm{R}$, et al., SIREN Study Group. SARS-CoV-2 infection rates of antibody-positive compared with antibody-negative healthcare workers in England: a large, multicentre, prospective cohort study (SIREN). Lancet. 2021;397(10283):1459-69. https://doi.org/10.1016/S0140-6736(21)00675-9 PMID: 33844963

11. Harris RJ, Hall JA, Zaidi A, Andrews NJ, Dunbar K, Dabrera $G$. Impact of vaccination on household transmission of SARS-COV-2 in England. Available from: https:// khub.net/documents/135939561/390853656/Impact +of+vaccination+on+household+transmission +of +SA RS-COV-2+in+England.pdf/35bf/bb1-6ade-d3eb-a3ge9c9b25a8122a?t=1619601878136 
12. Arora RK, Joseph A, Van Wyk J, Rocco S, Atmaja A, May E, et al. SeroTracker: a global SARS-CoV-2 seroprevalence dashboard. Lancet Infect Dis. 2021;21(4):e75-6. https://doi.org/10.1016/ S1473-3099(20)30631-9 PMID: 32763195

13. Population ages $0-14$ (\% of total population). Data. Washington DC: World Bank. [Accessed: 4 Apr 2021]. Available from: https://data.worldbank.org/indicator/SP.POP.0014.TO.ZS

14. Rodrigues EPS, Abreu IN, Lima CNC, da Fonseca DLM, Pereira SFG, Dos Reis LC, et al. High prevalence of anti-SARS-CoV-2 IgG antibody in the Xikrin of Bacajá (Kayapó) indigenous population in the brazilian Amazon. Int J Equity Health. 2021;20(1):50. https://doi.org/10.1186/s12939-021-01392-8 PMID: 33509206

15. Amante A. Over half of people tested in Italy's Bergamo have COVID-19 antibodies. The Jakarta Post. 2021. Available from: https://www.thejakartapost.com/news/2020/06/o9/over-halfof-people-tested-in-italys-bergamo-have-covid-19-antibodies. html

16. Hall V, Foulkes S, Charlett A, Atti A, Monk EJM, Simmons R, et al. Do antibody positive healthcare workers have lower SARS-CoV-2 infection rates than antibody negative healthcare workers? Large multi-centre prospective cohort study (the SIREN study), England: June to November 2020. medRxiv. 2021. 2021.01.13.21249642. https://doi.org/http://dx.doi.org/10.110 $1 / 2021.01 .13 .21249642$

17. Eggo RM, Dawa J, Kucharski AJ, Cucunuba ZM. The importance of local context in COVID-19 models. Nat. Comput. Sci.2021;1(1):6-8. https://doi.org/10.1038/ S43588-020-00014-7

18. Lovett S. UK secures 60 million additional Pfizer doses for autumn booster programme. The Independent. 2021. Available from: https://www.independent.co.uk/news/science/covidvaccine-pfizer-doses-booster-b1839074.html

19. Neil MF, Laydon D, Nedjati-Gilani G, Imai N, Ainslie K, Baguelin $M$, et al. Impact of non-pharmaceutical interventions (NPIs) to reduce COVID-19 mortality and healthcare demand. London: Imperial College London; 2020. https://doi.org/http://dx.doi. org/10.25561/77482

20. Office for National Statistics (ONS). Coronavirus (COVID-19) Infection Survey, UK, 5 March 2021.

Newport: ONS. 2021. Available from: https://www. ons.gov.uk/peoplepopulationandcommunity/ healthandsocialcare/conditionsanddiseases/bulletins/ coronaviruscovid19infectionsurveypilot/march2021

\section{License, supplementary material and copyright}

This is an open-access article distributed under the terms of the Creative Commons Attribution (CC BY 4.0) Licence. You may share and adapt the material, but must give appropriate credit to the source, provide a link to the licence and indicate if changes were made.

Any supplementary material referenced in the article can be found in the online version.

This article is copyright of the authors or their affiliated institutions, 2021. 